\title{
Inter-flap Distances in HIV-1 Protease Determined by Pulsed EPR Measurements
}

\author{
Luis Galiano $^{\S}$, Marco Bonora ${ }^{\dagger}$, Gail E. Fanucci ${ }^{{ }^{*}}$ \\ Department of Chemistry, University of Florida, PO Box 117200, Gainesville, Florida 32611 \\ $\dagger$ Institute of Molecular Biophysics, Florida State University, Tallahassee, Florida 32306 \\ fanucci@,chem.ufl.edu
}

\section{Further experimental Details:}

Protease Expression. A codon and expression optimized DNA sequence encoding the HIV-1 protease LAI consensus amino acid sequence was purchased from DNA2.0. This sequence also contained three mutations that provide protection from autocatolytic cleavage, Q7K, L33I, L63I, ${ }^{1}$ as well as mutations C67A and C95A to avoid unspecific spin labeling as well as disulfide crosslinking. ${ }^{2}$ We call this construct the pentamutated protease (PMPR) The gene was cloned to pET-23a vector (Novagen) under the control of the T7 promoter. Mutations D25N and K55C were introduced using the QuikChange sitedirected mutagenesis kit (Stratagene) and the sequence was confirmed by DNA sequencing. HIV-1 Protease mutants were expressed in BL21*(DE3) pLysS cells (Invitrogen) by adding $1 \mathrm{mM}$ isopropyl- $\beta$ D-thiogalactoside (IPTG) when the culture density (as determined by absorbance at $600 \mathrm{~nm}$ ) was 1.0 or greater. The expression and purification methodology was similar for PMPR and spin labeled cysteine mutant proteases. 
Protease Purification. Plasmid-encoded HIV-1 proteases were expressed as inclusion bodies in E. coli, as described in the literature ${ }^{3}$. In brief, cells were pelleted $\left(7500 \mathrm{~g}, 20 \mathrm{~min}, 4^{\circ} \mathrm{C}\right)$, resuspended in Buffer A (20 mM Tris, 1mM EDTA, $10 \mathrm{mM} \mathrm{BME} \mathrm{pH} \mathrm{7.5)} \mathrm{and} \mathrm{lysed} \mathrm{with} \mathrm{three} \mathrm{passes} \mathrm{through} \mathrm{a} 35 \mathrm{~mL}$ French pressure cell ( $\geq 16000$ psi) (Thermo Scientific). Cell debris and protease-containing inclusion bodies were collected by centrifugation $\left(18500 \mathrm{~g}, 25 \mathrm{~min}, 4^{\circ} \mathrm{C}\right)$. Inclusion bodies were washed in three steps using different buffers (Buffer 1: $25 \mathrm{mM}$ Tris, 2.5mM EDTA, 0.5M NaCl, $1 \mathrm{mM}$ diGly, $50 \mathrm{mM}$ BME, pH 7.0; Buffer 2: 25 mM Tris, 2.5 mM EDTA, $0.5 \mathrm{M} \mathrm{NaCl}, 1 \mathrm{mM}$ diGly, $50 \mathrm{mM}$ BME pH 7.0, 1 M Urea; Buffer 3: 25 mM Tris, 1 mM EDTA, 1 mM diGly, 50 mM BME, pH 7.0).

Each of the steps included washing, homogenization/sonication and centrifugation to collect inclusion bodies $\left(18500 \mathrm{~g}, 20 \mathrm{~min}, 4^{\circ} \mathrm{C}\right)$. HIV-1 protease inclusion bodies were solubilized ( $25 \mathrm{mM}$ TrisHCl, 5 $\mathrm{mM} \mathrm{NaCl}, 1 \mathrm{mM}$ EDTA, $1 \mathrm{mM}$ diGly, $50 \mathrm{mM}$ BME, 9 M Urea), clarified by centrifugation, (18500 $\mathrm{g}$, $25^{\circ} \mathrm{C}$ ), filtered through a $0.22 \mu \mathrm{M}$ filter, and applied directly to an anion-exchange Q-Sepharose column (HiTrap Q Sepharose HP, Amersham), previously equilibrated in the same buffer. Protease was collected as flowthrough; protease-containing fractions were pooled, and formic acid was added to a final concentration of $25 \mathrm{mM}$ to precipitate contaminants. Protease was stored overnight at $4^{\circ} \mathrm{C}$ and contaminants were removed by centrifugation $\left(39000 \mathrm{~g}, 4^{\circ} \mathrm{C}, 20 \mathrm{~min}\right)$.

HIV-1 protease was refolded by rapid dilution into $10 \mathrm{mM}$ formic acid $(\mathrm{pH} 3.0)$ at $0^{\circ} \mathrm{C}$. After refolding, the temperature was raised to $25^{\circ} \mathrm{C}$ and $\mathrm{pH}$ was increased to 5.0 by dropwise addition of 2.5 $\mathrm{M} \mathrm{NaOAc}(\mathrm{pH} 5.5)$. Precipitated contaminants were removed by centrifugation $\left(18500 \mathrm{~g}, 25 \mathrm{~min}, 4^{\circ} \mathrm{C}\right)$. Protease was concentrated and desalted (HiPrep 26/10 Desalting Column, Amersham)

Spin labeling. Cysteine mutants of HIV-1 protease were desalted into $10 \mathrm{mM}$ Tris, $\mathrm{pH} 6.9$ for the MTSL spin labeling reaction, and into $10 \mathrm{mM}$ HEPES, pH 7.6 for IAP. Spin labels were dissolved into $100 \% \mathrm{EtOH}$ and added to the protein solution in 100:1 label:protein concentration to ensure full 
labeling. Labeling reaction proceeded overnight at $4^{\circ} \mathrm{C}$. After labeling, spin labeled protease was desalted into $2 \mathrm{mM} \mathrm{NaOAc}$ buffer at $\mathrm{pH}$ 5.0. HIV protease was concentrated and stored at $\sim 100 \mu \mathrm{M}$ at $20^{\circ} \mathrm{C}$. The protease was estimated to be $\geq 99 \%$ pure by SDS-PAGE.

DEER experiments: Data were collected at the National High Magnetic Field Laboratory (NHMFL, Tallahassee) using a Bruker EleXsys E580/E680 equipped with the ER 4118X-MD5 Dielectric Ring Resonator under conditions of strong overcoupling at a temperature of $65 \mathrm{~K}$ at X-Band frequencies. The four-pulse DEER sequence $(\pi / 2)_{v 1}-\tau_{1}-(\pi)_{v 1}-t^{\prime}-(\pi)_{v 2}-\tau_{1}+\tau_{2}-t^{\prime}-(\pi)_{v 1}-\tau_{2}-e c h o$ was used, ${ }^{4}$ where the delay time , $t^{\prime}$, for the pump pulse $(\pi)_{v 2}$ is varied. The integrated intensity of the echo is recorded as a function of this delay time. For our experiments, data were typically recorded at steps of 12-16 ns, ranging from $0 \leq t^{\prime} \leq 3 \mathrm{~ms}$. The pulse lengths for $\pi / 2$ and $\pi$ were $16 \mathrm{~ns}$ and $32 \mathrm{~ns}$, respectively. The long interpulse delay, $\tau_{2}$ (typically $2-3 \mathrm{~ms}$ for our samples) was optimized for each sample by recording a spin-echo decay experiment and evaluating the phase memory time, $\mathrm{T}_{\mathrm{m}}$. In all cases, a $\tau_{1}$ of $200 \mathrm{~ns}$ was used. The pump frequency was set to the global maximum of the nitroxide spectrum (central peak) and the observer frequency was set to the relative maximum of the low field line (typically at a distance of 70-72 MHz from the central peak). Accumulation times for the different datasets varied between 2 - 24 hours, depending on protein concentration and interpulse delay.

Figures S1-S2 show time-domain dipolar evolution experimental data, solution for the optimal regularization parameter, distance distributions and L-curves for for HIV protease PMPR+D25N+K55C spin labeled with MTSL (S1) and IAP (S2). Tikhonov regularization was performed using the program DeerAnalysis2006. Exponential background was subtracted from the experimental signal, and the regularization parameter was obtained from regularization of the background subtracted echo curve. 
Regularization parameters were obtained using the L-curve criterion for which the optimal regularization parameter lies in the corner of the L-curve.

\section{PMPR+D25N+K55MTSL}
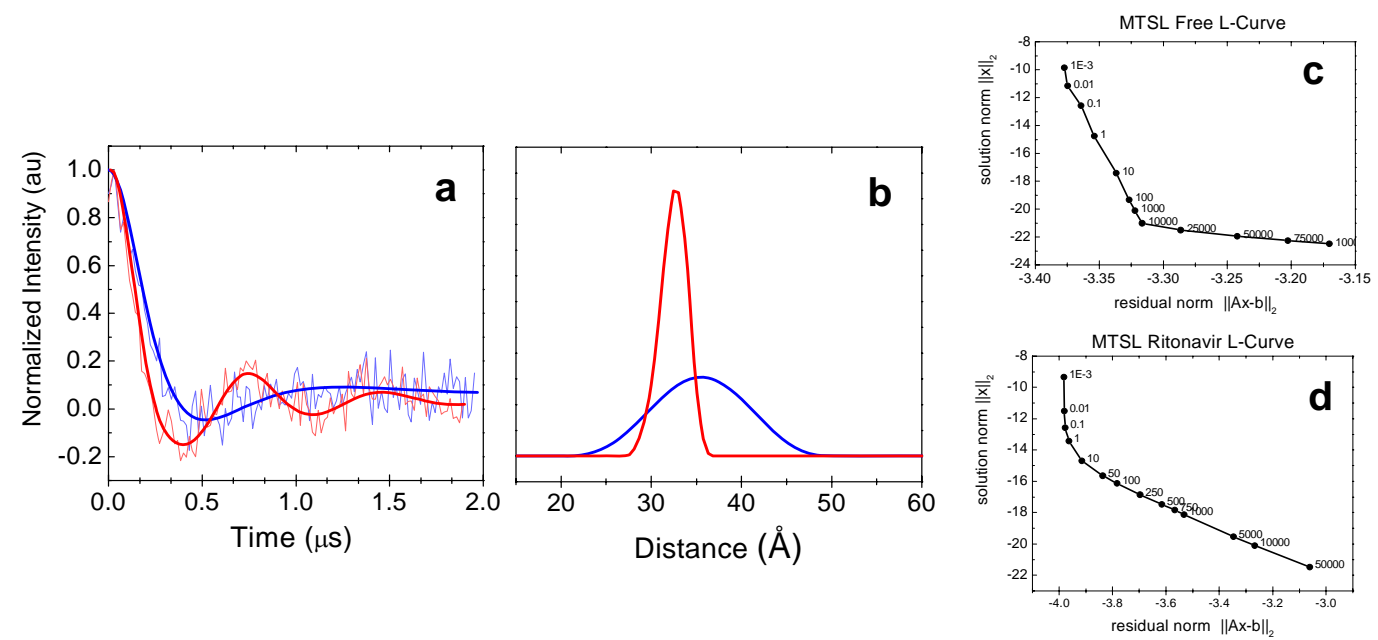

Figure S1: a) Normalized and background subtracted time domain dipolar evolution for PMPR+D25N+K55MTSL in presence (red) and absence (blue) of Ritonavir. The solid smooth lines through the experimental data are solutions obtained from Tikhonov Regularization (TKR). b) Distance distributions obtained by TKR for the time domain signals; presence (red) and absence (blue) of Ritonavir. The average distances are $35.5 \AA$ for the uninhibited form and $32.6 \AA$ for the inhibited one. (c-d). L-Curves used to choose the appropriate regularization parameter $\lambda$. In the absence of inhibitor, $\lambda=10000$ (c) and with Ritonavir, $\lambda=1(\mathrm{~d})$. In all cases, protein concentrations were $\sim 60-80 \mu \mathrm{M}$. For the inhibited sample, the ratio Protein:inhibitor was 1:4. 


\section{PMPR+D25N+K55IAP}
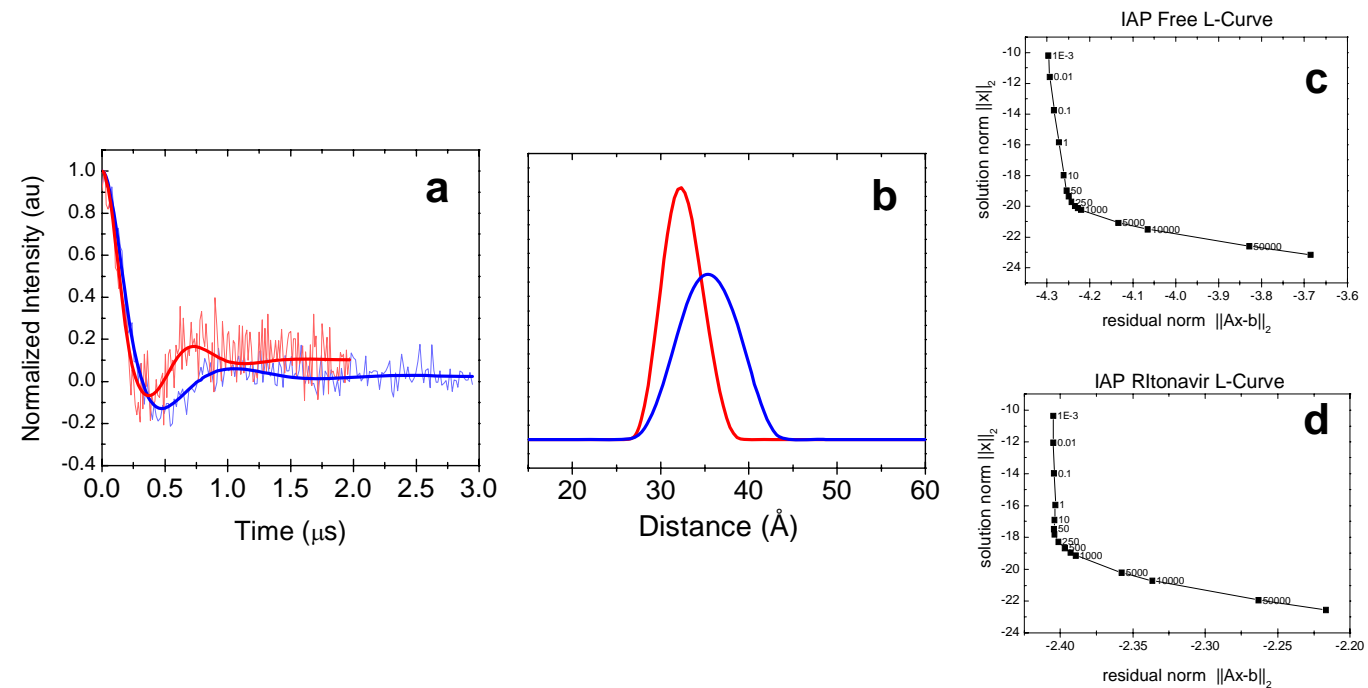

Figure S2: a) Normalized and background subtracted time domain dipolar evolution for PMPR+D25N+K55IAP in presence (red) and absence (blue) of Ritonavir. The solid smooth lines through the experimental data are solutions obtained from TKR b) Distance distributions obtained by TKR for the time domain signals; presence (red) and absence (blue) of Ritonavir. The average distances are $35.4 \AA$ for the uninhibited form and $32.4 \AA$ for the inhibited one. (c-d). L-Curves used to choose the appropriate regularization parameter $\lambda$. In the absence of inhibitor, $\lambda=250(\mathrm{c})$ and with Ritonavir, $\lambda=250(\mathrm{~d})$. In all cases, protein concentrations were $\sim 60-80 \mu \mathrm{M}$. For the inhibited sample, the ratio Protein:inhibitor was 1:4.

Comparison between Montecarlo and Tikhonov Regularization. Distance distributions and corresponding time-domain solutions for the optimal regularization parameter obtain by Tikhonov Regularization (TKR, Figs. S1-S2) were compared to those produced by a Montecarlo (MC) fitting approach. As it can be seen in figures S3-S6, the distance distribution profiles obtained using both data analysis approaches are almost identical. 
The small differences between both approaches arise from the use of Gaussian distributions for the MC fitting, as well as the small uncertainty for the choice of regularization parameter from the L-curve in TKR, making the use of both methods complementary to obtain an estimate on the error derived from the data fitting procedure.

\section{PMPR+D25N+K55MTSL}
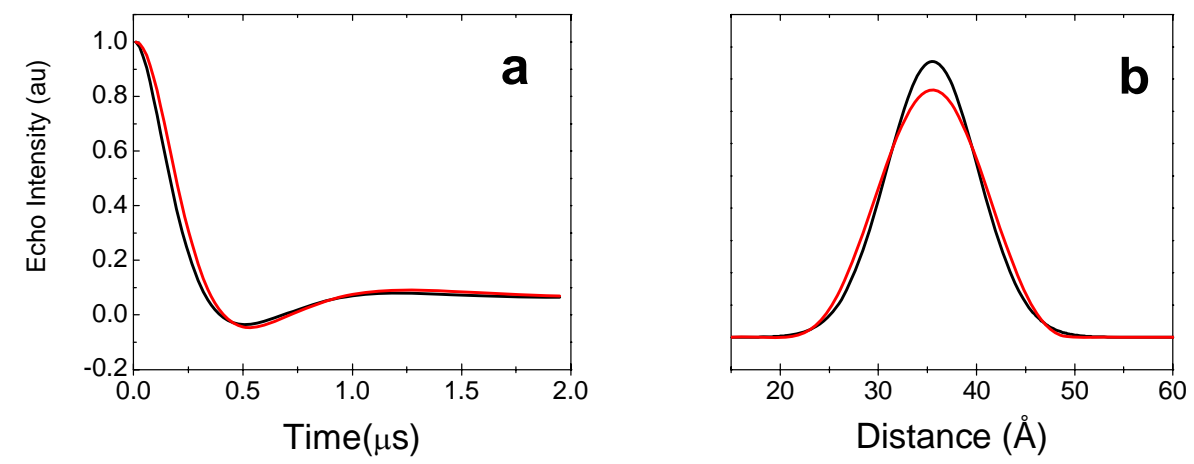

Figure S3: a) Normalized background subtracted time evolution signal corresponding to the best solutions for the echo decay of PMPR+D25N+K55MTSL from TKR (red) and MC (black) methods. b) Distance distribution profile solutions for the time evolution signals for TKR (red) and MC (black) methods.

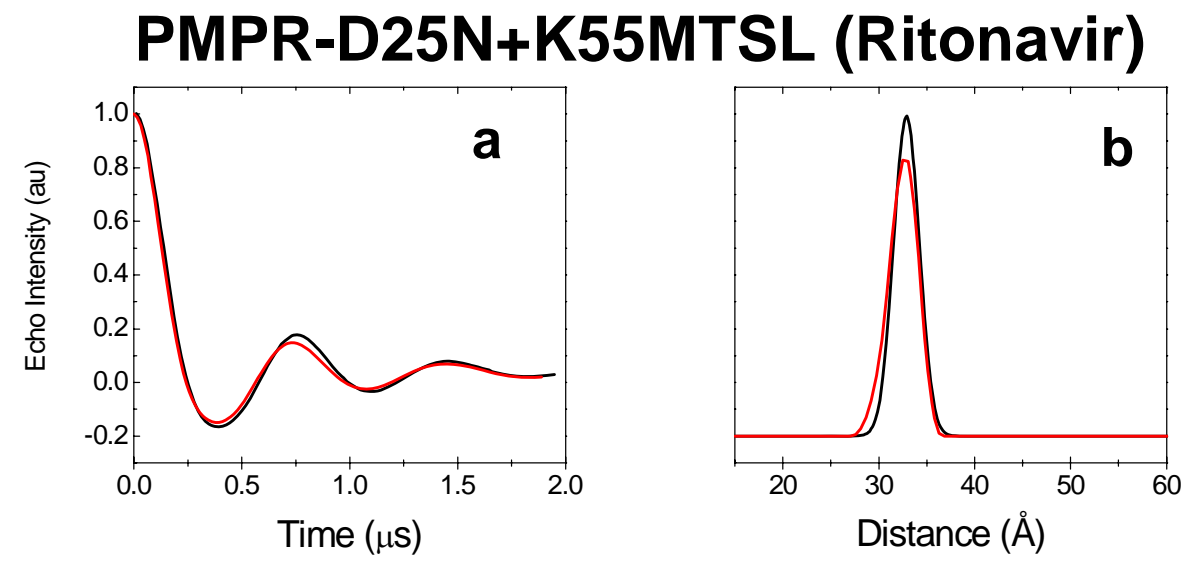

Figure S4: a) Normalized background subtracted time evolution signal corresponding to the best solutions for the echo decay of PMPR+D25N+K55MTSL (Ritonavir) using TKR (red) and MC (black) methods. b) Distance distribution profile solutions for the time evolution signals for TKR (red) and MC (black) methods. 


\section{PMPR+D25N+K55IAP}
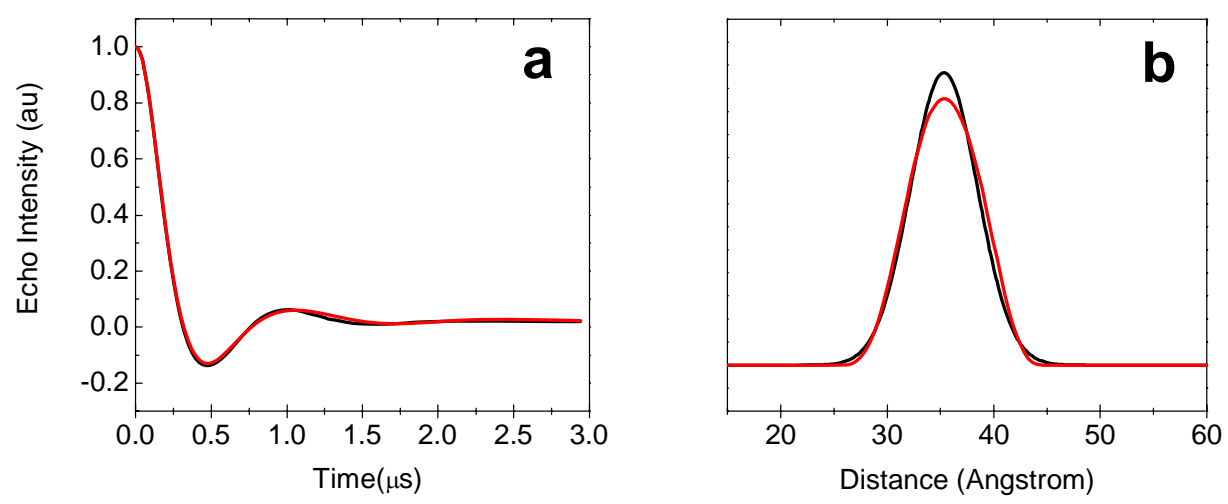

Figure S5: a) Normalized background subtracted time evolution signal corresponding to the best solutions for the echo decay of PMPR+D25N+K55IAP using TKR (red) and MC (black) methods. b) Distance distribution profile solutions for the time evolution signals for TKR (red) and MC (black) methods.

\section{PMPR+D25N+K55IAP (Ritonavir)}
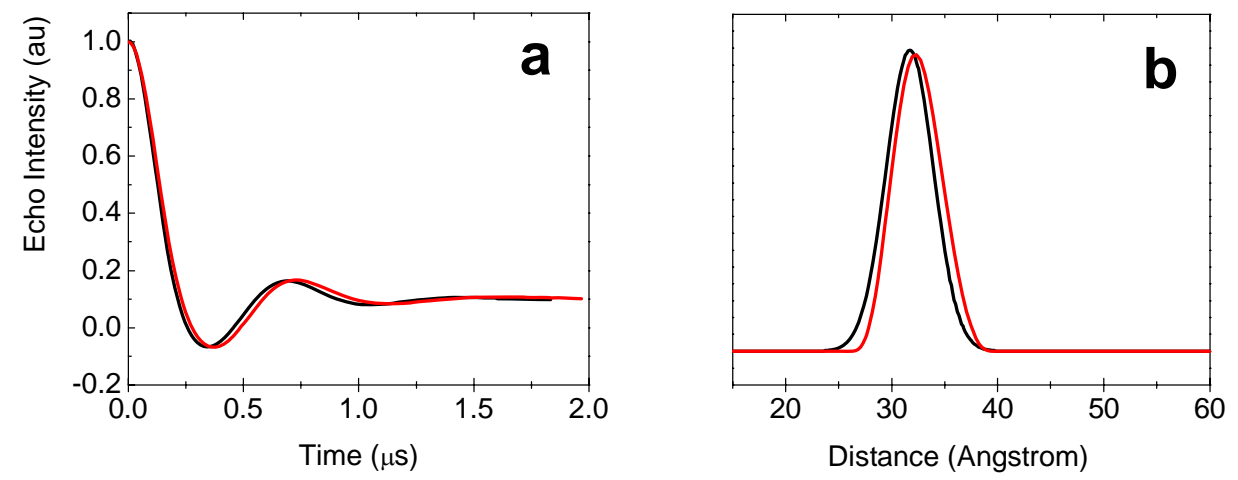

Figure S6: a) Normalized background subtracted time evolution signal corresponding to the best solutions for the echo decay of PMPR+D25N+K55IAP (Ritonavir) using TKR (red) and MC (black) methods. b) Distance distribution profile solutions for the time evolution signals for TKR (red) and MC (black) methods. 
Circular Dichroism. In order to ensure that the introduction of the Cys mutant and the spin label did not affect HIVPR structure, circular dichroism (CD) experiments were performed for the $\mathrm{PMPR}+\mathrm{D} 25 \mathrm{~N}+\mathrm{K} 55 \mathrm{C}$ mutant as well as PMPR+D25N+K55MTSL. As can be seen in Figure $\mathrm{S} 4$, the CD spectra of the CYS mutant and SL construct are nearly identical to that reported in the literature for the LAI consensus sequence, indicating that the mutation at $\mathrm{K} 55 \mathrm{C}$ and spin labeling do not significantly alter the structure of the protease.

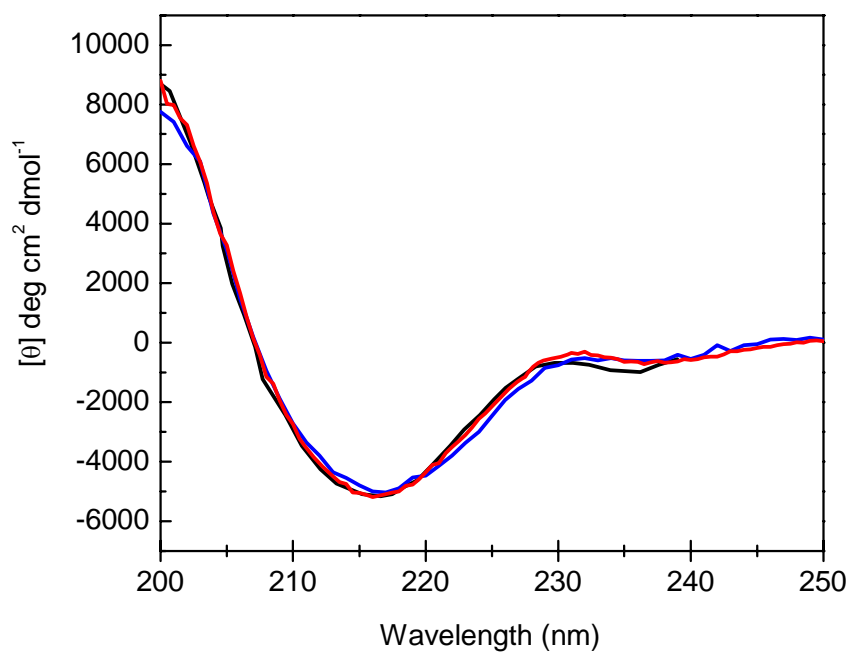

Figure S7: Ditigized Circular dichroism spectrum for HIV protease LAI consensus sequence (active) taken from the literature ${ }^{5}$ (black line), PMPR $+\mathrm{D} 25 \mathrm{~N}+\mathrm{K} 55 \mathrm{C}$ (blue line) and PMPR+D25N+K55MTSL (red line). Measurements were collected in $20 \mathrm{mM} \mathrm{NaOAc} \mathrm{pH} 4.5$ at $\sim 0.3 \mathrm{mg} / \mathrm{mL}$ protein concentration as determined by absorption at $280 \mathrm{~nm}$, using an extinction coefficient of $1.15 \mathrm{mg} \mathrm{cm}^{-1} \mathrm{ml}^{-1}$

Protease inhibition assay. Although the protein used for the EPR experiments has the active site mutation, D25N, we include here the results of an inhibition assay on PMPR $+\mathrm{K} 55 \mathrm{SL}$ as evidence that the change in experimental conditions necessary for spin labeling do not alter or affect the ability of the active protein to cleave substrate or bind to Ritonavir. The inhibition assay validates that these changes in experimental conditions needed for spin labeling (compared to preparation of samples for X-ray 
crystallography or NMR measurements) do not affect the protein function. As such the catalytic activity of PMPR+K55SL (both spin labels were tested) was monitored following the hydrolysis of chromogenic substrate VI (Lys-Ala-Arg-Val-Nle-nPhe-Glu-Ala-Nle-NH $\mathrm{NH}_{2}$ ).(American Peptide Company, Inc.) ${ }^{6}$ This substrate resembles the cleavage site ARVL/AEAM between the capsid and p2 polypeptide sequences in the gag polyprotein of HIV virus.

Protease was added to a $125 \mu \mathrm{L}$ microcuvette (Varian, Inc) containing substrate at $37^{\circ} \mathrm{C}$. Final concentrations in the assay buffer were: $50 \mathrm{mM} \mathrm{NaOAc} \mathrm{pH} 4.7,150 \mathrm{mM} \mathrm{NaCl}, 4 \mathrm{mM}$ EDTA, $100 \mu \mathrm{M}$ substrate, $\sim 40 \mathrm{nM}$ concentration of protein. The absorbance was monitored at nine wavelengths (296$304 \mathrm{~nm}$ ) using a Varian Cary 50 spectrometer (Varian Inc.) equipped with a peltier water bath and a 18cell holder, and corrected for spectrometer drift by subtracting the absorbance at 446-454 nm. ${ }^{7}$

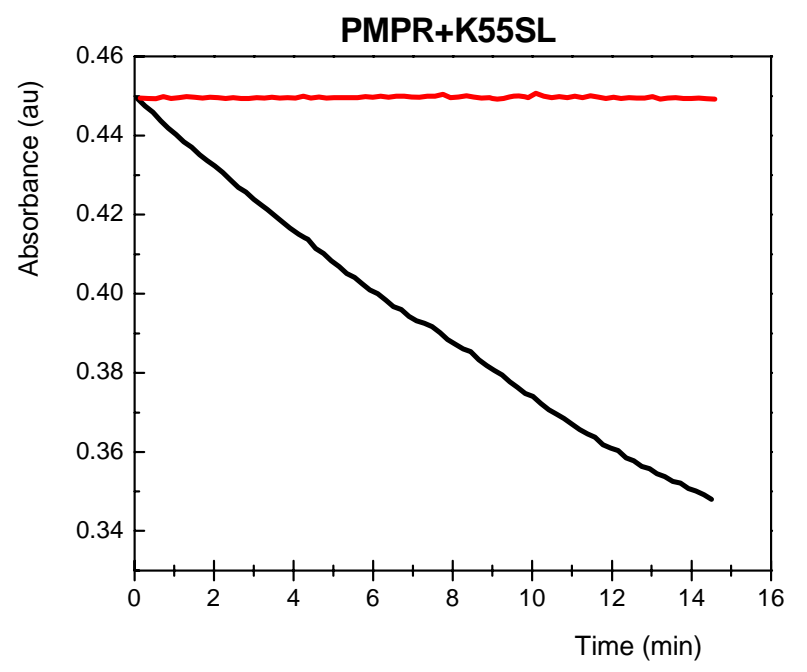

Figure S8. Chromogenic substrate degradation followed by UV/Vis. In black, PMPR+K55SL and in red, PMPR+K55SL+Ritonavir. It can be easily seen that there is full inhibition of the protease upon addition of inhibitor (Ritonavir); hence, the presence of the spin-label is not interfering with inhibitor binding of protease function. Full kinetic characterization of the protease is beyond the scope of this manuscript and will be included in forthcoming work. Concentrations were $\sim 40 \mathrm{nM}$ protein, $100 \mu \mathrm{M}$ chomogenic substrate. For the inhibition, Ritonavir was added in 4:1 Ritonavir:Protease excess to mimic the conditions used in the pulsed EPR experiments. 


\section{References}

1. Mildner, A. M.; Rothrock, D. J.; Leone, J. W.; Bannow, C. A.; Lull, J. M.; Reardon, I. M.; Sarcich, J. L.; Howe, W. J.; Tomich, C. S.; Smith, C. W.; et al., Biochemistry 1994, 33, (32), 9405-13.

2. $\quad$ Louis, J. M.; Clore, G. M.; Gronenborn, A. M., Nat Struct Biol 1999, 6, (9), 868-75.

3. $\quad$ Ohtaka, H.; Schon, A.; Freire, E., Biochemistry 2003, 42, (46), 13659-66.

4. $\quad$ Pannier, M.; Veit, S.; Godt, A.; Jeschke, G.; Spiess, H. W., J Magn Reson 2000, 142, (2), 33140.

5. Xie, D.; Gulnik, S.; Gustchina, E.; Yu, B.; Shao, W.; Qoronfleh, W.; Nathan, A.; Erickson, J. W., Protein Sci 1999, 8, (8), 1702-7.

6. Phylip, L. H.; Richards, A. D.; Kay, J.; Kovalinka, J.; Strop, P.; Blaha, I.; Velek, J.; Kostka, V.; Ritchie, A. J.; Broadhurst, A. V.; et al., Biochem Biophys Res Commun 1990, 171, (1), 439-44.

7. Dunn, B. M.; Scarborough, P. E.; Davenport, R.; Swietnicki, W., Methods Mol Biol 1994, 36, 225-43. 